Z. Klin. Chem. Klin. Biochem.

13. Jg. 1975, S. 79-84

\title{
Methodische Untersuchungen zur Liquorelektrophorese
}

\author{
Von H.-D. Krause, H. Wisser und K. M. Pirke \\ Aus dem Institut für Klinische Chemie (Geschäftsführender Direktor: Professor Dr. Dr. J. Büttner) der Medizini- \\ schen Hochschule Hannover und der Abteilung für Klinische Chemie des Max-Planck-Institutes für Psychiatrie \\ (Geschäftsfiihrender Direktor: Professor Dr. D. Ploog) München
}

(Eingegangen am 13. September/4. Dezember 1974)

An normalen und pathologischen Liquorproben sowie an Modelliquores wurden methodische Untersuchungen zur Elektrophorese durchgefürt. Es wurden drei Einengverfahren - Ultrafiltration, Vakuumfil tration und Druckfiltration - verglichen. Bei der Vakuum- und Ultrafiltration wurden höhere Albumin- und geringere $\boldsymbol{\gamma}$-Globulinwerte gemessen als bei der Druckfiltration. Für keines der Einengverfahren wurde eine Abhängigkeit der Meßwerte von der Proteinkonzentration und dem Probevolumen gefunden.

Ein Vergleich von Amidoschwarz 10 B- und Ponceau S-Färbung ergab signifikant höhere Albuminwerte für die Ponceau S-Färbung. Für alle anderen Fraktionen wurden höhere Werte nach Anfärbung mit Amidoschwarz 10 B gefunden.

Der Einfluß der Probeverwahrung bei $-20^{\circ} \mathrm{C}$ wurde untersucht. Innerhalb von 17 Tagen wurden keine Veränderungen beobachtet. Nach 6-monatiger Verwahrung wurden mit Ausnahme eines deutlichen Anstiegs der $\boldsymbol{\gamma}$-Globulinfraktion nur sehr geringfügige Veränderungen gemessen.

\section{Methodological studies on the electrophoretic analysis of cerebrospinal fluid}

Methodological studies are reported on the electrophoretic analysis of normal and pathological cerebrospinal fluid, and of model fluids. Three concentration methods were compared - ultrafiltration, vacuum filtration and high pressure filtration. After vacuum and ultrafiltration the albumin values were higher and the $\gamma$-globulin values were lower than after high pressure filtration. No dependence of the measured values on the protein concentration or the sample volume was observed for any of the concentration methods.

After staining with Ponceau $S$ the values for albumin were significantly higher than after staining with Amido black 10B.

The effect of storing samples at $-20^{\circ} \mathrm{C}$ was investigated. There were no observable changes over a period of 17 days. After 6 months storage, the $\gamma$-globulin fraction showed a marked increase, but otherwise there were only slight changes.

Zur elektrophoretischen Auftrennung der Liquoreiweiße ist wegen des niedrigen Gesamteiweißgehaltes eine vorherige Konzentrierung erforderlich. Dazu kommen routinemäßig verșchiẹdene Methoden zur Anwendung (1-6). Durch Verluste beim Konzentrationsvorgang oder Denaturierung der Eiweiße kann es bei den einzelnen Verfahren zu Fehlern unterschiedlicher Ausprägung kommen $(2,7)$. Es sind auch Verfahren beschrieben, die eine Liquorelektrophorese ohne Konzentrierung ermöglichen $(8,9)$. Ein Ziel dieser Arbeit ist es, durch Vergleich von drei verschiedenen Konzentrierungsverfahren eine Methode zu finden, die mit kleinem Probevolumen auskommt und von ungeübtem Personal mit reproduzierbaren Ergebnissen durchführbar ist. Gleichzeitig sollte der Einfluß von unterschiedlichen Anfärbemethoden auf die einzelnen Proteinfraktionen untersucht werden.

Uber den Störeinfluß der Probeverwahrung auf das Gesamteiweiß und die einzelnen Fraktionen nach elektrophoretischer Trennung liegen keine systematischen
Untersuchungen vor. Rieder (10) weist in einer kürzlich erschienenen Arbeit darauf hin, daß bei längerem Aufbewahren die Liquorproteine unkontrollierbare Veränderungen erfahren. Daher wurde in einer weiteren Versuchsreihe der Einfluß dieses Parameters untersucht.

\section{Methodik}

\section{Konzentrierung der Liquorproben}

Druckfiltration

Hinsichtlich methodischer Einzelheiten sei auf die Publikation von Mertin et al. verwiesen (11).

Für die Druckfiltration wurden Ultrafilter Typ SM 121/36 ${ }^{1}$ ) benutzt. Nach Angaben des Herstellers erfolgt die Retention der Eiweiße ab einem Molekulargewicht von 10000.

\section{Ultrafiltration}

Für die Anreicherung durch Ultrafil tration wurde das CentrifloUltrafil trationssystem ${ }^{2}$ ) (Centriflo-Ultrafil ter-GF 50) benutzt.

\footnotetext{
1) Sartorius-Membranfilter $\mathrm{GmbH}$, Göttingen

$\left.{ }^{2}\right)$ Fa. Amicon N. V., Oosterhout N.B., Holland
} 
Für dic Zentrifugation wurden dic Zentrifugenbecher mit Kunststoffeinsätzen versehen, die die Aufnahme der „centrifuge tube“ ermöglichten. Die Einengung wurde bei $2000 \mathrm{U} / \mathrm{min}$ durchgeführt, wobei für etwa $0,5 \mathrm{ml}$ Liquor 10 Minuten benötigt wurden. Mit eincr $20 \mu \mathrm{l}$-Pipette wurde der konzentrierte Liquor entnommen. Anschließend wurden die Ultrafilter mit bidest. Wasser mehrfach gespült, bei Raumtemperatur getrocknet und wieder verwendet. Die Eiweißretention erfolgt ab einem Molekulargewicht von 50000 .

\section{Vakuumfiltration}

Bei diesem Verfahren wurde eine Vakuumfiltrationsvorrichtung mit Kollodiumhülsen ${ }^{1}$ ) benutzt. Vor Gebrauch müssen die in Methanol aufbewahrten Kollodiumhülsen für mindestens 60 Minuten in dest. Wasser gelegt werden. Anschließend werden diese etwa $1 \mathrm{~cm}$ auf eine Kunststoffmanschette des Einsatzrohres gezogen und in das mit bidest. Wasser oder $\mathrm{NaCl}-\mathrm{Lösung}$ $(0,15 \mathrm{~mol} / \mathrm{l})$ gefüllte Auffanggefäß eingesetzt. Nach Einbringen der Probe in die Kollodiumhülse erfolgt die Konzentrierung bei einem Druck von etwa 500 Torr. Da die Kollodiumhülsen transparent sind, läßt sich der Einengvorgang beobach ten. Die Konzentrierung wurde bis zu einem Restvolumen von 10 bis $20 \mu$ l durchgefürt. Die Retention der Eiweiße erfolgt nach Angaben des Herstellers ab einem Molekulargewicht von 12400.

\section{Elektrophorese}

Die elektrophoretische Trennung der Eiweiße wurde auf Celluloseacetat-Folien $\left.(25,5 \times 145 \mathrm{~mm})^{3}\right)$ in einer Mikrophor-Elektrophoresekammer mit dem Netzgerät Pherostat ${ }^{4}$ ) durchgeführt. Als Puffer-, Färbe-, Entfärbe- und Transparenzlösung wurden Fertiglösungen der Firma Boskamp benuitzt. Die Auswertung erfolgte mit dem Extinktionsschreiber 3 mit Integralschreiber ${ }^{5}$ ).

\section{Gesamteiweißbestimmung}

Die Bestimmung des Gesamteiweißes wurde mit der BiuretMethode nach Fällung des Proteins mit 20proz. Trichloressigsäure durchgefuihrt.

\section{Ergebnisse und Diskussion}

\section{Vergleich der verschiedenen Einengverfahren}

Durch Verdünnen eines Kontrollserums $(1: 200)$ wurde ein ,Modell-Liquor“ hergestellt. In einer Vorperiode wurden die Sollkonzentrationen des unverdünnten Kontrollserums der einzelnen Eiweißfraktionen ermittelt. Die Anfärbung erfolgte mit Amidoschwarz 10 B. Zur Konzentrierung wurde $1 \mathrm{ml}$ des „Modell-Liquors“ eingesetzt. Bei der Vakuumfiltration werden normalerweise größere Probevolumina eingesetzt.

Bei den in Tabelle 1 wiedergegebenen Ergebnissen fällt auf, daß nach Konzentrierung durch Vakuumfiltration die Albuminwerte deutlich höher und die $\alpha_{2^{-}}, \beta$ - und $\gamma$-Globulinwerte niedriger liegen. Bei den beiden anderen Methoden ist die Übereinstimmung für die Albumine und $\gamma$-Globuline mit den Sollwerten gut. Dies ist insofern unerwartet, da nach den von den Herstellerfirmen angegebenen Retentionswerten der Eiweiße Druck- und Vakuumfiltration zu etwa gleichen Ergebnissen hätten führen sollen, vorausgesetzt, daß dieser Parameter von starkem Einfluß ist.

\footnotetext{
3) Fa. Schleicher \& Schüll, Dassel, Krs. Einbeck

$\left.{ }^{4}\right) \mathrm{Fa}$. Boskamp, Hersel b. Bonn

5) Fa. Carl Zeiss, Oberkochen/Württ.
}

Tab. 1. Präzision der einzelnen Anreicherungsverfahren bei einem konstanten Probevolumen von $1,0 \mathrm{ml}$ und einer Proteinkonzentration von $345 \mathrm{mg} / \mathrm{l}(\mathrm{n}=6)$. Die in der obersten Zeile in Klammern angegebenen Werte sind Sollwerte der einzelnen Fraktionen, die in einer Vorperiode unter Benutzung des unverdünnten Serums bestimmt wurden.

\begin{tabular}{|c|c|c|c|c|c|c|}
\hline \multirow[b]{2}{*}{$\begin{array}{l}\text { Eineng- } \\
\text { verfahren }\end{array}$} & \multirow{2}{*}{$\begin{array}{l}\text { statist. } \\
\text { Kenn- } \\
\text { größe } \\
\overline{\mathrm{X}} \text { rel. \% }\end{array}$} & \multirow{2}{*}{$\begin{array}{l}\text { Albu- } \\
\text { min } \\
(59,0)\end{array}$} & \multicolumn{4}{|c|}{ Globuline } \\
\hline & & & $\begin{array}{l}\alpha_{1} \\
(3,5)\end{array}$ & $\begin{array}{l}\alpha_{2-}^{-} \\
(10 ; 5)\end{array}$ & $\begin{array}{l}\beta- \\
(12,5)\end{array}$ & $\begin{array}{l}r \\
(14,5)\end{array}$ \\
\hline $\begin{array}{l}\text { Druck- } \\
\text { filtration }\end{array}$ & $\begin{array}{l}\overline{\mathrm{x}} \text { rel. \% } \\
\text { s rel. \% } \\
\text { V \% }\end{array}$ & $\begin{array}{c}62,8 \\
2,04 \\
3,3\end{array}$ & $\begin{array}{l}4,3 \\
0,41 \\
9,4\end{array}$ & $\begin{array}{l}8,9 \\
0,74 \\
8,3\end{array}$ & $\begin{array}{l}9,8 \\
0,76 \\
7,8\end{array}$ & $\begin{array}{c}14,1 \\
1,24 \\
8,8\end{array}$ \\
\hline $\begin{array}{l}\text { Ultra- } \\
\text { filtration }\end{array}$ & $\begin{array}{l}\overline{\mathrm{x}} \text { rel. \% } \\
\text { s rel. \% } \\
\text { V \% }\end{array}$ & $\begin{array}{c}60,2 \\
1,57 \\
2,6\end{array}$ & $\begin{array}{l}5,2 \\
0,41 \\
7,9\end{array}$ & $\begin{array}{l}9,3 \\
1,08 \\
11,6\end{array}$ & $\begin{array}{c}10,8 \\
0,68 \\
6,3\end{array}$ & $\begin{array}{c}14,3 \\
0,52 \\
3,6\end{array}$ \\
\hline $\begin{array}{l}\text { Vakuum- } \\
\text { filtration }\end{array}$ & $\begin{array}{l}\bar{x} \text { rel. \% } \\
\text { s rel. \% } \\
\text { V \% }\end{array}$ & $\begin{array}{c}66,3 \\
1,21 \\
1,8\end{array}$ & $\begin{array}{l}3,9 \\
0,20 \\
5,2\end{array}$ & $\begin{array}{l}8,5 \\
0,55 \\
6,4 .\end{array}$ & $\begin{array}{l}9,4 \\
0,38 \\
4,0\end{array}$ & $\begin{array}{c}11,9 \\
0,58 \\
4,9\end{array}$ \\
\hline
\end{tabular}

In einer zweiten Versuchsreihe sollte geprüft werden, ob das Volumen oder die Eiweißkonzentration als Parameter bei den verschiedenen Einengverfahren Einfluß haben. Daher wurden unterschiedliche Volumina verdünnter Serumproben mit einer konstanten Proteinkonzentration von $345 \mathrm{mg} / \mathrm{l}$ und konstante Volumina bei unterschiedlicher Proteinkonzentration zur Konzentrierung angesetzt. Die Ergebnisse dieser Versuchsreihe sind in Tabelle 2 zusammengefaßt.

Diese Meßergebnisse zeigen keinen Einfluß des Probevolumens oder der Eiweißkonzentration nach Konzentrierung durch Druck- und Ultrafiltration. Bei der Vakuumfiltration ist nach den vorliegenden Werten ein Einfluß der Eiweißkonzentration anzunehmen. Auch bei dieser Versuchsreihe liegen für die Vakuumfiltration die Werte der Albuminfraktion höher und die der $\boldsymbol{\gamma}$-Globulinwerte niedriger. Ergebnisse an verdünnten Serumproben sind nicht ohne weiteres übertragbar auf Liquorproben. Daher wurden 16 verschiedene Liquorproben mit den drei verschiedenen Konzentrierungsverfahren eingeengt. und anschließend die elektrophoretische Trennung durchgeführt. Da es sich hierbei um normale und pathologische Proben handelte (Gesamteiweißgehalt zwischen 280 und $1070 \mathrm{mg} / \mathrm{l}$ ), wurden die mittlere Abweichung der einzelnen Fraktionen nach Konzentrierung mittels Ultra- und Vakuumfiltration gegenüber der Druckfiltration berechnet (Tab. 3).

Die Ergebnisse der Poolliquorproben zeigen gegenüber dem Ergebnis der Serumproben insofern eine Abweichung, als bei den Liquorproben Vakuum- und Ultrafiltration niedrigere Albumin- und höhere $\boldsymbol{\gamma}$-Globulinwerte liefern. Die absoluten Abweichungen sind zwar nicht sehr groß, aber statistisch signifikant (KruskalWallis-Test, $\mathrm{p}<0,05$ ).

Wenn auch nach den Ërgebnissen von Tabelle 1 die Präzision nach Konzentrierung mittels Druckfiltration 
Tab. 2. Einfluß von Probevolumen und Gesamtproteinkonzentration auf die Ergebnisse der Elektrophorese in Abhängigkeit von drei verschiedenen Einengverfahren (Mittelwerte von $n=3$ ).

\begin{tabular}{|c|c|c|c|c|c|c|c|c|c|c|c|c|}
\hline $\begin{array}{l}\text { Eineng- } \\
\text { verfahren }\end{array}$ & $\begin{array}{l}\text { Probe- } \\
\text { volumen } \\
(\mathrm{ml})\end{array}$ & $\begin{array}{l}\text { Albumin } \\
\text { rel. \% }\end{array}$ & $\begin{array}{l}\text { Globuline } \\
\alpha_{1}- \\
\text { rel. } \%\end{array}$ & $\begin{array}{l}\alpha_{2-}^{-} \\
\text {rel. } \%\end{array}$ & $\begin{array}{l}\beta- \\
\text { rel. } \%\end{array}$ & $\underset{\text { rel. } \%}{\gamma}$ & $\begin{array}{l}\text { Protein } \\
\text { konz. } \\
\text { (mg/l) }\end{array}$ & $\begin{array}{l}\text { Albumin } \\
\text { rel. \% }\end{array}$ & $\begin{array}{l}\text { Globuline } \\
\alpha_{1-}^{-} \\
\text {rel. } \%\end{array}$ & $\begin{array}{l}\alpha_{2}^{-} \\
\text {rel. } \%\end{array}$ & $\begin{array}{l}\beta \\
\text { rel. } \%\end{array}$ & $\stackrel{\gamma}{r}$ rel. $\%$ \\
\hline $\begin{array}{l}\text { Druck- } \\
\text { filtration } \\
(345 \mathrm{mg} / \mathrm{l})\end{array}$ & $\begin{array}{l}0,5 \\
1,0 \\
1,5 \\
\end{array}$ & $\begin{array}{l}64 \\
64 \\
62 \\
\end{array}$ & $\begin{array}{l}3,5 \\
4,5 \\
3,3 \\
\end{array}$ & $\begin{array}{l}7,7 \\
6,5 \\
8,2 \\
\end{array}$ & $\begin{array}{r}10,2 \\
9,5 \\
10,7 \\
\end{array}$ & $\begin{array}{l}15 \\
15 \\
17 \\
\end{array}$ & $\begin{array}{l}690 \\
345 \\
173 \\
\end{array}$ & $\begin{array}{l}61,5 \\
62,3 \\
62,3 \\
\end{array}$ & $\begin{array}{l}3,8 \\
4,5 \\
4,0 \\
\end{array}$ & $\begin{array}{l}8,8 \\
8,5 \\
7,8\end{array}$ & $\begin{array}{r}10,7 \\
9,5 \\
10,5\end{array}$ & $\begin{array}{l}15,2 \\
15,2 \\
15,3\end{array}$ \\
\hline $\begin{array}{l}\text { Ultra- } \\
\text { filtration } \\
(345 \mathrm{mg} / \mathrm{l})\end{array}$ & $\begin{array}{l}0,5 \\
1,0 \\
1,5\end{array}$ & $\begin{array}{l}66 \\
66 \\
65\end{array}$ & $\begin{array}{l}3,3 \\
3,0 \\
3,0 \\
\end{array}$ & $\begin{array}{l}5,5 \\
5,7 \\
5,8 \\
\end{array}$ & $\begin{array}{l}8,8 \\
9,3 \\
9,0 \\
\end{array}$ & $\begin{array}{l}16 \\
16 \\
17 \\
\end{array}$ & $\begin{array}{l}690 \\
345 \\
173 \\
\end{array}$ & $\begin{array}{l}62,5 \\
66,2 \\
64,3\end{array}$ & $\begin{array}{l}3,0 \\
3,0 \\
3,2\end{array}$ & $\begin{array}{l}6,8 \\
5,7 \\
5,2\end{array}$ & $\begin{array}{r}9,7 \\
9,3 \\
10,2\end{array}$ & $\begin{array}{l}18,0 \\
15,8 \\
16,8\end{array}$ \\
\hline $\begin{array}{l}\text { Vakuum- } \\
\text { filtration } \\
(345 \mathrm{mg} / \mathrm{l})\end{array}$ & $\begin{array}{l}0 ; 5 \\
1,0 \\
1,5\end{array}$ & $\begin{array}{l}65 \\
68 \\
67\end{array}$ & $\begin{array}{l}3,5 \\
3,3 \\
2,8\end{array}$ & $\begin{array}{l}7,3 \\
6,8 \\
6,0\end{array}$ & $\begin{array}{r}10,5 \\
9,2 \\
9,0\end{array}$ & $\begin{array}{l}13,5 \\
12,3 \\
14,8\end{array}$ & $\begin{array}{l}690 \\
345 \\
173\end{array}$ & $\begin{array}{l}61,5 \\
68,3 \\
73,2\end{array}$ & $\begin{array}{l}3,5 \\
3,3 \\
3,0\end{array}$ & $\begin{array}{l}8,0 \\
6,8 \\
5,3\end{array}$ & $\begin{array}{r}10,7 \\
9,2 \\
8,3\end{array}$ & $\begin{array}{l}16,3 \\
12,3 \\
10,5\end{array}$ \\
\hline
\end{tabular}

Tab. 3. Mittlere Differenzen der verschiedenen Fraktionen nach Konzentrierung mit Ultrafil tration bzw. Vakuumfiltration gegenüber der Druckfiltration bei 16 verschiedenen Liquorproben.

\begin{tabular}{lcccc}
\hline Fraktion & \multicolumn{2}{c}{ Ultrafil tration } & \multicolumn{2}{c}{ Vakuumfiltration } \\
\hline & $\overline{\mathrm{d}}($ rel. \%) & $s_{\mathrm{d}}($ rel. \%) & $\overline{\mathrm{d}}$ (rel. \%) & $\mathrm{s}_{\mathrm{d}}$ (rel. \%) \\
Präalbumin & 0,27 & 0,61 & $-0,22$ & 0,57 \\
Albumin & $-4,70$ & 3,48 & $-2,03$ & 4,61 \\
$\alpha_{1}$-Globulin & 0,81 & 0,66 & 0,16 & 0,82 \\
$\alpha_{2}$-Globulin & 0,11 & 0,81 & 0,98 & 1,21 \\
$(\beta+\tau)$-Globulin & $-0,59$ & 1,95 & 1,39 & 1,58 \\
$\gamma$-Globulin & 2,58 & 1,96 & 1,23 & 1,08 \\
\hline
\end{tabular}

schlechter ist als bei den übrigen Verfahren, so haben wir uns doch wegen der einfachen Handhabung für diese Methode entschieden. Sie ist von ungeübten Kräften leichter zu erlernen und gegenüber der Vakuumfiltration schneller durchführbar, da der Einengvorgang je nach Probevolumen $(100-500 \mu \mathrm{l})$ nach 10 bis 30 Minuten beendet ist. Vorteilhaft wirkt sich bei der Druckfiltration aus, daß man bei Gesamteiweißkonzentrationen über $500 \mathrm{mg} / \mathrm{l}$ mit $100 \mu \mathrm{l}$ Liquor auskommen kann. Dadurch verkürzt sich die.Einengzeit auf etwa 10 Minuten und vermindert dadurch möglicherweise die Gefahr der Denaturierung. Ein Nachteil der Ultrafiltration ist, daß bei zu langer Z̈entrifugation die einżuengende Probe ganz durch das Filter gelaufen ist. Es gelingt dann nicht mehr, z.B. durch Zugabe von Puffer, Eiweiß für die elektrophoretische Trennung zu gewinnen. Die gleiche Membran kann allerdings mehrmals benutzt werden. Dies konnte durch wechselweise Konzentrierung eines verdünnten Kontrollserums und Plasmocytomserums nachgewiesen werden.

\section{Einfluß des Färbemittels}

Bei der Versuchsreihe zur Probeverwahrung von Liquorproben (s. unten) wurde die Färbung nach Konzentrierung durch Druckfiltration und elektrophoretischer
Trennung mit Amidoschwarz 10 B und Ponceau S durchgefuihrt. Die dabei untersuchte Stichprobe umfaßt 92 verschiedene Probanden und enthält normale und pathologische Proben. Da der dabei untersuchte Konzentrationsbereich relativ groß ist und somit auch die Streuungen, wurden die Mittelwerte der einzelnen Fraktionen bei unterschiedlicher Anfärbung und deren Streuung ermittelt. (Tab. 4) Ein positiver Wert der mittleren Differenz bedeutet, daß die Werte für die Anfärbung mit Amidoschwarz 10 B durchschnittlich höher liegen als die nach Anfärbung mit Ponceau S. Die statistische Auswertung wurde wie folgt durchgeführt: Nur diejenigen Differenzen zwischen der Anfärbung mit Amidoschwarz 10 B und Ponceau S wurden als von Null verschieden betrachtet, die mindestens gleich \pm 1 sind. Diese Differenzen wurden mit Hilfe des Vorzeichentestes geprüft.

Betrachtet man zunächst die mittleren Differenzen, so sieht man, daß die Absolutwerte relativ klein sind. Mit Ausnahme des Albumins sind alle Differenzen positiv, d.h., die „Amidoschwarz 10 B-Werte" liegen höher als die „Ponceau S-Werte“. Die statistische Auswertung (einseitiger Test) und die entsprechenden Testschwellen ergeben für die Ponceau S-Färbung signifikant kleinere Albuminwerte. Für alle anderen Fraktionen liegen die

Tab. 4. Durch unterschiedliche Färbemittel (Amidoschwarz 10 B und Ponceau $S$ ) bedingte mittlere Differenzen der einzelnen Eiweißfraktionen, Streuung dieser Differenzen sowie die Testschwellen (Druckfiltration, $n=92$ ).

\begin{tabular}{lccc}
\hline Fraktion & $\overline{\mathrm{d}}(\mathrm{rel} . \%)$ & $\mathrm{s}_{\mathrm{d}}$ (rel.\%) & Testschwelle \\
\hline Präalbumin & 0,28 & 0,93 & $0,57 \%$ \\
Albumin & $-2,56$ & 4,61 & $4 \cdot 10^{-6} \%$ \\
$\alpha_{1}$-Globulin & 0,84 & 1,07 & $1 \cdot 10^{-8} \%$ \\
$\alpha_{2}$-Globulin & 0,56 & 0,95 & $7 \cdot 10^{-4} \%$ \\
$(\beta+\tau)$-Globulin & 0,42 & 1,78 & $12,2 \%$ \\
$\gamma$-Globulin & 0,46 & 2,16 & $0,34 \%$ \\
\hline
\end{tabular}


Verhältnisse umgekehrt. Allerdings sind die Differenzen bei $\operatorname{der}(\beta+\tau)$-Fraktion statistisch nicht signifikant $(\mathrm{p}<0,05)$. Bei der Anfärbung mit Ponceau $S$ fehlte außerdem die $\tau$-Fraktion häufiger, so daß sich für die Liquorelektrophorese die Anfärbung mit Amidoschwarz 10 B empfiehlt.

\section{Probeverwahrung}

Uber die Frage der Probeverwahrung von Liquorproben gibt es wenige Untersuchungen. Rieder et al. (10) erwähnen in einer Publikation, daß Liquorproteine bei längerem Aufbewahren unkontrollierbare Veränderungen erfahren.

In einer ersten Versuchsreihe untersuchten wir Poolliquorproben in Doppelbestimmungen an 17 aufeinanderfolgenden Arbeitstagen. Poolliquor wurde in 1,5 mlPortionen in Mikroreaktionsgefäße abgefüllt und dann bis zur Analyse bei $-20^{\circ} \mathrm{C}$ aufbewahrt. Das Ergebnis dieser Untersuchung ist in Tabelle 5 wiedergegeben. Zum Vergleich sind die Ergebnisse für eine verdünnte und unverdünnte Kontrollprobe mitangegeben, wobei letztere ohne Konzentrierung analysiert wurde.

Vergleicht man das Ergebnis der Poolliquorproben und das der verdünnten Serumkontrollprobe mit dem der unverdünnten Kontrollprobe, so zeigt sich, daß die Absolutwerte der Streuungen in etwa übereinstimmen, d.h. der Einfluß der Konzentrierung auf die Streuung ist nicht groß. Trägt man die Einzelwerte in Abhängigkeit vom Zeitpunkt der Messung auf, so läßt sich, wie aus Abbildung 1 ersichtlich ist, für das Albumin und $\gamma$-Globulin beim Poolliquor keine eindeutige zunehmende oder abnehmende Tendenz erkennen.

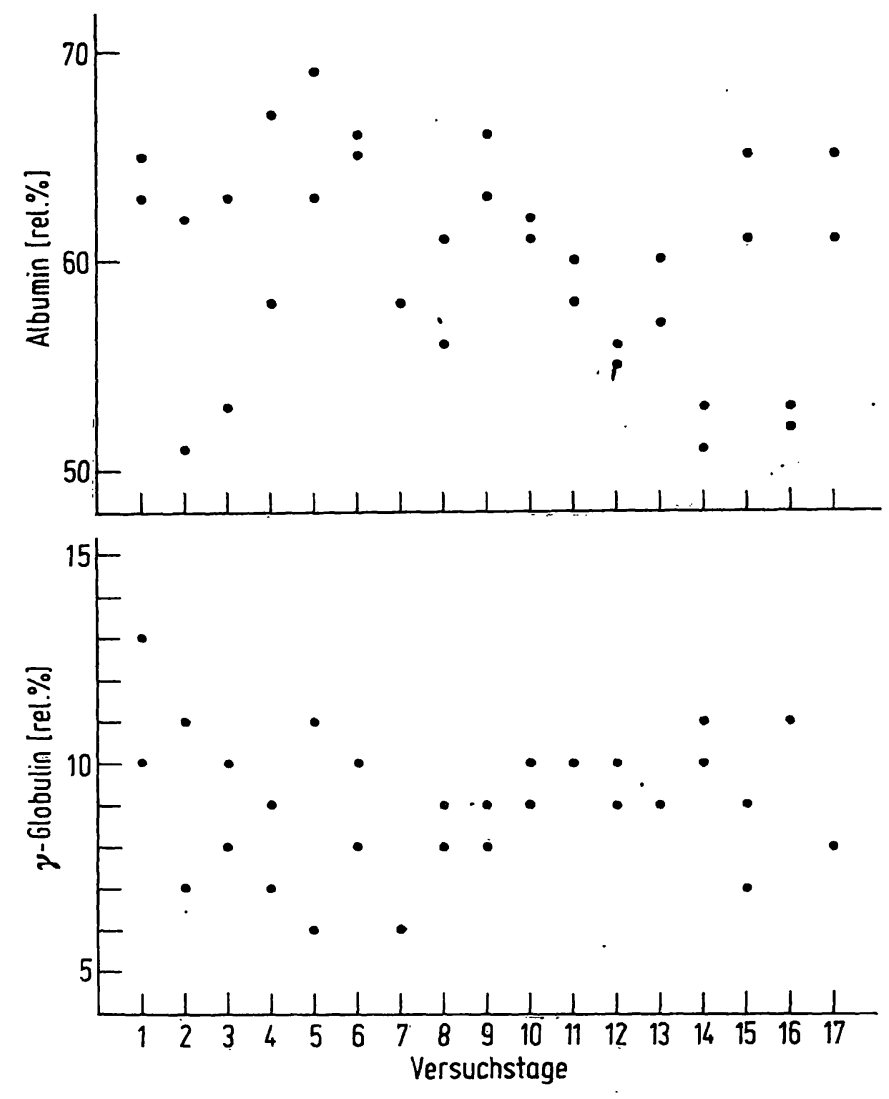

Abb. 1. Albumin- und $\gamma$-Globulin-Werte eines Poolliquors in Abhängigkeit von der Zeit (Doppelbestimmungen).

Dieser Befund stützt zunächst die Annahme, daß für einen kurzen Zeitraum eine Probeverwahrung möglich ist.

Tab. 5. Reproduzierbarkeit der Bestimmung der verschiedenen Eiweißfraktionen nach elektrophoretischer Trennung (Konzentrierung: Druckfiltration, Färbung: Amidoschwarz $10 \mathrm{~B}$ ).

\begin{tabular}{|c|c|c|c|c|c|c|c|c|}
\hline Probe & Streuung & $\begin{array}{l}\text { statistische } \\
\text { Kenngröße }\end{array}$ & Präalbumin & Albumin & $\begin{array}{l}\text { Globuline } \\
\alpha_{1^{-}}\end{array}$ & $\alpha_{2}^{-}$ & $\beta-(b \bar{z} w . \beta+\tau)$ & $\boldsymbol{\gamma}$ \\
\hline \multirow[t]{2}{*}{ Poolliquor } & $\begin{array}{l}\text { in der } \\
\text { Serie } \\
(n=16)\end{array}$ & $\begin{array}{l}\bar{x} \text { rel \% } \\
\text { s rel. \% } \\
\text { V \% }\end{array}$ & $\begin{array}{r}5,2 \\
0,8 \\
15,7\end{array}$ & $\begin{array}{r}60,0 \\
3,7 \\
6,1\end{array}$ & $\begin{array}{r}5,3 \\
0,9 \\
16,2\end{array}$ & $\begin{array}{r}6,9 \\
1,1 \\
16,4\end{array}$ & $\begin{array}{r}13,6 \\
1,5 \\
11,4\end{array}$ & $\begin{array}{r}9,1 。 \\
1,6 \\
17,6\end{array}$ \\
\hline & $\begin{array}{l}\text { von Tag } \\
\text { zu Tag } \\
(n=17)\end{array}$ & $\begin{array}{l}\bar{x} \text { rel. \% } \\
\text { s rel. \% } \\
\text { V \% }\end{array}$ & $\begin{array}{r}5,3 \\
0,9 \\
16,4\end{array}$ & $\begin{array}{r}59,6 \\
4,8 \\
8,1 \\
\end{array}$ & $\begin{array}{r}5,5 \\
1,3 \\
22,7\end{array}$ & $\begin{array}{r}6,9 \\
1,6 \\
23,3\end{array}$ & $\begin{array}{r}13,7 \\
2,8 \\
20,2\end{array}$ & $\begin{array}{r}9,1 \\
1,9 \\
21,1\end{array}$ \\
\hline \multirow[t]{2}{*}{$\begin{array}{l}\text { Kontrollprobe } \\
1: 100 \text { verdünnt } \\
\text { (Serum) }\end{array}$} & $\begin{array}{l}\text { in der } \\
\text { Serie } \\
(n=15)\end{array}$ & $\begin{array}{l}\overline{\mathbf{x}} \text { rel. \% } \\
\mathrm{s} \text { rel. \% } \\
\mathrm{V} \%\end{array}$ & $\begin{array}{l}- \\
-\end{array}$ & $\begin{array}{r}69,0 \\
2,4 \\
3,4\end{array}$ & $\begin{array}{r}3,0 \\
0,4 \\
14,2\end{array}$ & $\begin{array}{r}6,3 \\
0,8 \\
12,5\end{array}$ & $\begin{array}{r}7,9 \\
0,9 \\
11,3\end{array}$ & $\begin{array}{r}13,6 \\
1,1 \\
8,3\end{array}$ \\
\hline & $\begin{array}{l}\text { von Tag } \\
\text { zu Tag } \\
(n=15)\end{array}$ & $\begin{array}{l}\overline{\mathbf{x}} \text { rel. \% } \\
\text { s rel. \% } \\
\text { V \% }\end{array}$ & $\begin{array}{l}- \\
-\end{array}$ & $\begin{array}{r}69,0 \\
3,5 \\
5,1\end{array}$ & $\begin{array}{r}3,0 \\
0,4 \\
12,6\end{array}$ & $\begin{array}{r}6,3 \\
1,4 \\
22,9\end{array}$ & $\begin{array}{r}7,8 \\
1,4 \\
18,3\end{array}$ & $\begin{array}{r}13,6 \\
1,4 \\
9,9\end{array}$ \\
\hline \multirow[t]{2}{*}{$\begin{array}{l}\text { Kontrollprobe } \\
\text { unverdünnt } \\
\text { (Serum) }\end{array}$} & $\begin{array}{l}\text { in der } \\
\text { Serie } \\
(n=16)\end{array}$ & $\begin{array}{l}\overline{\mathbf{x}} \text { rel. \% } \\
\text { s rel. \% } \\
\text { V \% }\end{array}$ & $\begin{array}{l}- \\
-\end{array}$ & $\begin{array}{r}66,1 \\
1,5 \\
2,2\end{array}$ & $\begin{array}{r}3,6 \\
0,9 \\
23,4\end{array}$ & $\begin{array}{r}7,8 \\
1,1 \\
14,0\end{array}$ & $\begin{array}{l}9,6 \\
0,8 \\
8,2\end{array}$ & $\begin{array}{r}12,9 \\
1,1 \\
8,2\end{array}$ \\
\hline & $\begin{array}{l}\text { von Tag } \\
\text { zu Tag } \\
(n=16)\end{array}$ & $\begin{array}{l}\overline{\mathbf{x}} \text { rel. \% } \\
\text { s rel. \% } \\
\text { V \% }\end{array}$ & $\begin{array}{l}- \\
-\end{array}$ & $\begin{array}{r}66,3 \\
-\quad 2,1 \\
3,2\end{array}$ & $\begin{array}{r}3,4 \\
0,7 \\
21,2\end{array}$ & $\begin{array}{r}8,1 \\
1,1 \\
13,4\end{array}$ & $\begin{array}{r}9,4 \\
1,3 \\
13,4\end{array}$ & $\begin{array}{r}12,8 \\
1,5 \\
11,8\end{array}$ \\
\hline
\end{tabular}



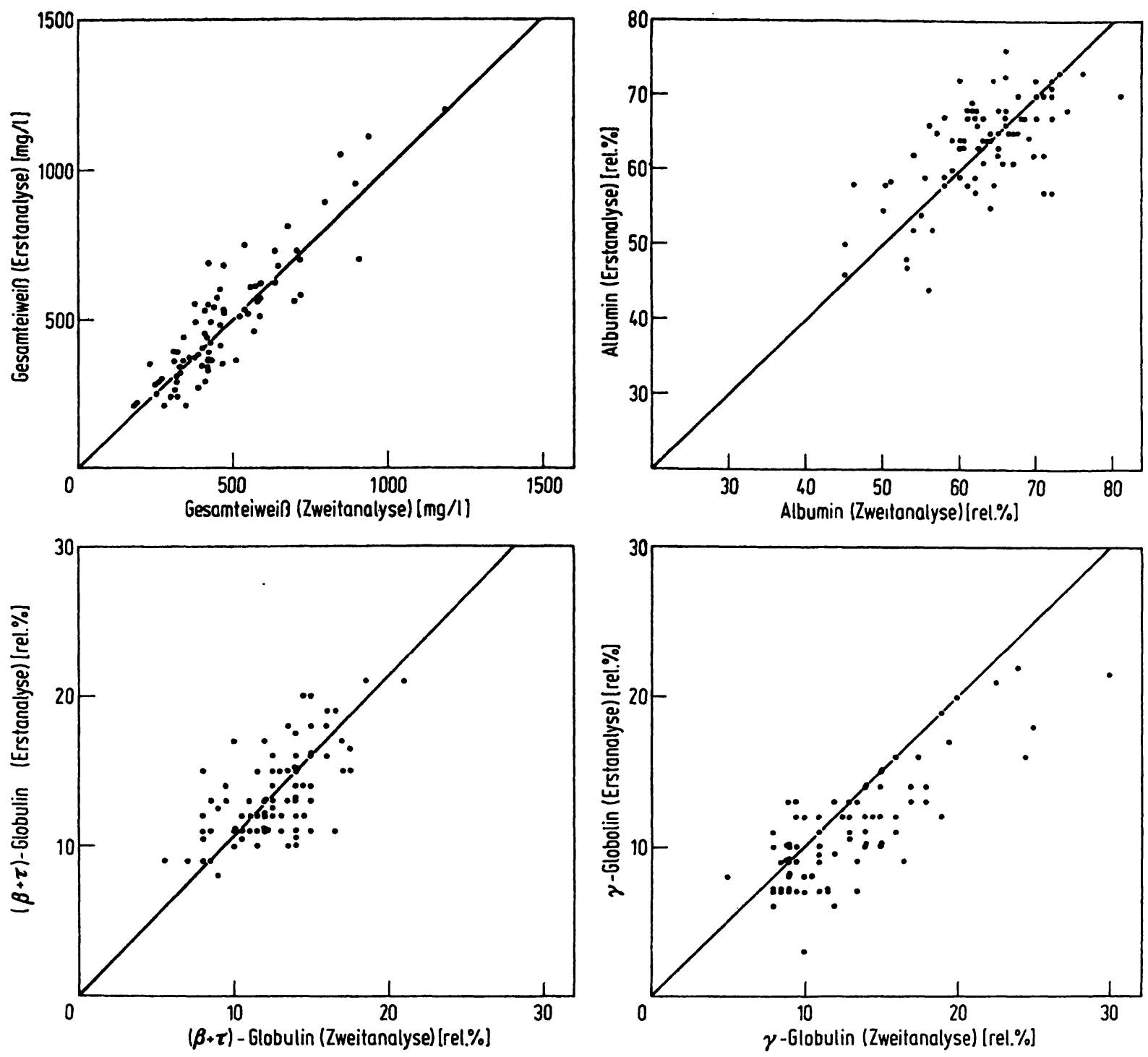

Abb. 2. Einfluß der Probeverwahrung von Liquorproben auf das Ergebnis der Bestimmung von Gesamteiweiß, Albumin, $(\beta+\tau)$-und $r$-Globulin.

Tab. 6. Einfluß der Probeverwahrungszeit auf das Gesamteiweiß und die elektrophoretischen Fraktionen. Angegeben sind die mittleren Abweichungen, deren Streuung und Testschwellèn, sowie die Korrelationskoeffizienten der Erstanalyse zur Zweitanalyse (Aufbewahrungszeit $6 \mathrm{Mon}$. bei $-20^{\circ} \mathrm{C}, \mathrm{n}=82$ ).

\begin{tabular}{|c|c|c|c|c|c|c|c|}
\hline & Gesamteiweiß & Präalbumin & Albumin & $\begin{array}{l}\text { Globuline } \\
\alpha_{1^{-}}\end{array}$ & $\alpha_{2}-$ & $\beta+\tau-$ & $r$ \\
\hline & $(\mathrm{mg} / \mathrm{l})$ & rel. $\%$ & rel. \% & rel. \% & rel. \% & rel. $\%$ & rel. \% \\
\hline $\bar{d}$ & $+20,1$ & $+0,43$ & $+0,73$ & $-0,48$ & $+0,12$ & 0,84 & $-1,63$ \\
\hline $\mathbf{s}$ & 96,4 & 1,06 & 5,66 & 1,28 & 1,34 & 2,51 & 3,07 \\
\hline Testschwelle & $>5 \%$ & $1 \%$ & $>5 \%$ & $0,5 \%$ & $>5 \%$ & $2,5 \%$ & $0,5 \%$ \\
\hline $\mathbf{r}$ & 0,973 & 0,733 & 0,684 & 0,353 & 0,493 & 0,635 & 0,856 \\
\hline
\end{tabular}

In einer zweiten Versuchsreihe wurden 83 Liquorproben (normal/pathologisch) nach der Analyse 6 Monate bei $-20^{\circ} \mathrm{C}$ aufbewahrt und nach Auftauen bei $+4^{\circ} \mathrm{C}$ erneut analysiert (Konzentrierung: Druckfịltration, Anfärbung: Amidoschwarz 10 B). Für das Gesamteiweiß sowie die Fraktionen Albumin, $(\beta+\tau)$-Globuline und $\gamma$-Globulin ist das Ergebnis in Abbildung 2 wiedergegeben.
Für das $\gamma$-Globulin ist aus dieser Abbildung eine $\mathrm{Zu}$ nahme der Werte bei der Zweitanalyse gegenüber der Erstanalyse zu beobachten. Für die anderen Bestandteile ist aus diesen Abbildungen keine eindeutige Tendenz in der einen oder anderen Richtung abzuleiten. Die statistische Auswertung der untersuchten Stichprobe ist in Tabelle 6 zusammengefaßt. 
Angegeben sind die mittlere Abweichung ( $\overline{\mathrm{d}})$, deren Streuung s, die Testniveaus der mit dem Vorzeichentest ausgewerteten Untersuchungsreihen, sowie die Korrelationskoeffizienten r. Ein negatives Vorzeichen bei den mittleren Differenzen bedeutet niedrigere Werte bei der Zweitanalyse. Wie man sieht, sind die mittleren Differenzen sehr klein. Mit Ausnahme des Gesamteiweißes, des Albumins und $\alpha_{2}$-Globulins sind diese statistisch signifikant. Die Frage, ob diesen statistisch signifikanten Unterschieden eine reale Bedeutung zukommt, dürfte nicht einfach zu entscheiden sein. Zumindest scheint beim $\gamma$-Globulin (s. auch Abb. 2) eine deutliche Abhängigkeit von der Verwahrungszeit zu bestehen. Die unterschiedliche Größe der Korrelationskoeffizienten wurde erwartet, denn bei der recht großen methodischen Streuung der Elektrophorese sind z.B. bei den kleinen Fraktionen keine hohen Korrelationen möglich. Zusammenfassend kann gesagt werden, daß bei einer kurzfristigen Probeverwahrung keine Abweichungen gefunden wurden, bei einer längerfristigen dagegen solche nicht mit Sicherheit auszuschließen sind.

\section{Danksagung}

Wir danken Fräulein Christa Aumann für ihre gewissenhafte Mitarbeit. Für die statistische Auswertung danken wir Herrn Dr. E. Hansert, Abteilung für Biometrie des MPI für Psychiatrie, München.

\title{
Literatur
}

1. Maier, K. H. \& Voggel, K. (1963).Klin. Wochenschr. 41, 286-290.

2. Delank, H. W. (1965) Das Eiweißbild des Liquor cerebrospinalis, Dr. Dietrich Steinkopf Verlag, Darmstadt.

3. Burrows, St. (1965) Clin. Chem. 11, 1068-1069.

4. Kaplan, W. \& Johnstone, M. (1966) Clin. Chem. 12, 717727.

5. Kaplan, A. \& Savory, J. (1970) Stand. Meth. Clin. Chem. VI, 13-30. Academic Press, New York and London.

6. Windisch, R. M. \& Braeken, M. M. (1970) Clin. Chem. 16, 416-419.

7. Kleine, T. O., Stroh, Maria \& Stroh, J. (1974) diese Z. 12, 66-72.

8. Rice, J. D. \& Bleakney, B. (1965) Clin. Chim. Acta, 12, 343-348.

9. Kleiné, T. O. \& Stroh, J. (1974) diese Z. 12, 73-80.

10. Rieder, H. P., Jung, P. R. \& Burri, V. (1972) diese Z. 10, 379-384.

11. Mertin, J.; Wisser, H. \& Doerr, P. (1971) diese Z. 9, 337-340.

\author{
Dr. H.-D. Krause \\ Zentrallabor der \\ Ev. Diakonissen-Anstalt \\ 6720 Spey.er \\ Hilgardstraße 26 \\ Priv. Doz. Dr. Dr. H. Wisser \\ Abteilung für Klinische Chemie \\ Robert-Bosch-Krankenhaus \\ 7000 Stuttgart 50 \\ Auerbachstraße 110
}

